Website: http://revistas.lamolina.edu.pe/index.php/acu/index

(C) Universidad Nacional Agraria La Molina, Lima - Perú

\title{
Rendimiento de pepino (Cucumis sativus L.) en función del tipo de bandeja y la edad de transplante de las plántulas
}

\section{Cucumber (Cucumis sativus L.) yield in function of tray type and seedling age at transplanting}

\author{
Nelson José Montaño Mata; José Alexander Gil Marín²*; Yeniledys Palmares² \\ *Autor de correspondencia
}

\section{Resumen}

Se estudió el efecto del volumen de sustrato y de la edad de las plántulas en la producción de pepino 'Poinsett 76' en bajo condiciones de campo en el sector de San Vicente, Maturín, estado Monagas, Venezuela, de abril a junio (2011). Los tratamientos consistieron en cuatro volúmenes de sustrato $\left(52,25,18\right.$ y $11 \mathrm{~cm}^{3}$ en bandeja plástica) y dos edades de plántulas ( 7 y 14 días después de la siembra). El diseño experimental fue en bloques al azar, con cuatro repeticiones y treinta plantas por parcela. Fueron evaluaron la cosecha precoz, intermedia, tardía y total. Las variables longitud, diámetro, peso del fruto, número de frutos por planta, rendimiento por planta y por hectárea. Para las condiciones de este experimento, se concluye que la producción total fue superior en las plántulas producidas en bandejas con mayor volumen de sustrato ( 52 y $25 \mathrm{~cm}^{3}$, respectivamente). Sin embargo, las plántulas producidas en menor volumen de sustrato presentaron menor rendimiento cuando ellas fueron más viejas (14 días). La edad influyo en la producción total de frutos cuando las plántulas fueron trasplantadas a los siete días después de la siembra. La edad no influyo sobre la longitud, diámetro, peso del fruto, número de frutos por planta en las plántulas producidas en volumen de sustrato mayor. La producción de plántulas de pepino en bandejas resulto en alta productividad (con media de 91,3 $\mathrm{t}^{*} \mathrm{ha}^{-1}$ ).

Palabras clave: Cucumis sativus; tipo de bandeja; rendimiento; pepino; tamaño de celda.

\begin{abstract}
The effect of the cell size and seedling transplanting age on the production of cucumber 'Poinsett 76' under field cultivation was studied, from April to June (2011) in Maturin, Monagas State, Venezuela. The treatments resulted from the combination of four tray cell sizes $\left(52,25,18\right.$ and $\left.11 \mathrm{~cm}^{3}\right)$ and two seedlings transplanting ages (seven and 14 days after sowing), evaluated in a randomized block design, with four replications and thirty plants per plot. During the harvest period were evaluated the harvest precocious, late, intermediate, and total yield. Fruit length, fruit diameter, fruit weight, number of fruits per plant, yield per plant, and yield of fruit per hectare were evaluated. The best results were obtained with the seedlings produced in greater volume of substrate trays. To the conditions of this experiment, it was ended that total production was improved when seedlings were produced in larger sized trays (52 and $25 \mathrm{~cm}^{3}$ respectively). However, seedling produced in smaller cell volume had their yield minor when they were older (14 day). Transplanting age had influence over the total yield of fruits when seedlings were seven days after sowing transplanting. Transplanting age had no influence on the fruit length, fruit diameter, fruit weight, number of fruits per plant when seedlings were obtained in large sized trays. The seedlings produced in trays resulted in high yield (average of $91,3 \mathrm{t}^{*} \mathrm{ha}^{-1}$ )
\end{abstract}

Keywords: Cucumis sativus; tray type; yield; cucumber; cell size.

\section{Introducción}

Dentro de las hortalizas tropicales, las cucurbitáceas ocupan un lugar destacado, siendo sus frutos de amplia aceptación popular. El pepino (Cucumis sativus L.) es una de las especies más cultivadas en Venezuela después de la patilla y melón, debido a su importancia socioeconómica, en la generación de empleos directos en la región de siembra, principalmente cuando el cultivo es realizado en el sistema de empalado, además de presentar alto valor nutricional. En el último año, el cultivo de pepino se colocó entre las principales cucurbitáceas producidas en Venezuela, con una superficie cosechada de 1248 hectáreas, registrándose un rendimiento por hectáreas de $16.017 \mathrm{t}^{*} \mathrm{ha}^{-1}$, y una producción de 19989 toneladas (Fedeagro, 2017). La dependencia de las semillas importadas de variedades e híbridos de altos costos y la inestabilidad del ciclo de precipitación en el país y en el estado Monagas, como consecuencia del cambio climático que se ha observado a nivel mundial, sumado a la necesidad de incrementar la producción de alimentos, ha llevado a la búsqueda de alternativas para enfrentar las dificultades que se presentan al momento de la siembra. Actualmente, se está expandiendo el uso de bandejas plásticas por los agricultores con el propósito de obtener las plántulas para

\footnotetext{
${ }^{1}$ Departamento de Agronomía

${ }^{2}$ Departamento de Ingeniería Agrícola, Núcleo Monagas, Universidad de Oriente (UDO). Avenida Universidad Campus Los Guaritos, Maturín, 6201, estado Monagas, Venezuela y, UDO, Maturín. Email: jalexgil2005@hotmail.com.
} 
el trasplante en campo. Ha llegado al mercado venezolano, y en especial en el estado Monagas semillas de híbridos de pepino con precio superior a los de cultivares. Debido al mayor costo, los agricultores vienen utilizando bandejas para producción de plántulas. Actualmente, en la producción de plántulas, se utilizan bandejas con celdas de varios tamaños y para maximizar la producción de plántulas en vivero, los productores utilizan celdas con el menor volumen posible. Según, Minami (1995), en las bandejas hay mejor aprovechamiento de las semillas, produciendo cada semilla una plántula. Comparada a la siembra directa, con el uso de esa tecnología ocurre una disminución en las fallas de pegado inicial en el campo, también como aumento en la uniformidad inicial de las plantas. Pero la reducción del volumen puede afectar la calidad de la plántula en consecuencia, la productividad y calidad del producto. La restricción radical, observada en pequeños volúmenes de celdas, puede perjudicar el crecimiento y la fotosíntesis, el contenido de clorofila en las hojas, la absorción de nutrientes y agua, la respiración, la floración, también la producción (Nesmith y Duval, 1998). Otro factor importante que puede afectar la calidad y productividad de las plantas, es la edad de las plántulas para el trasplante. Si la planta es mantenida por un periodo muy largo en la bandeja, pudiera ocurrir deficiencia de nutrientes y reducción de su calidad. El tiempo en que las plántulas permanezcan con su sistema radical restringido debe ser el mínimo posible (Pereira y Martínez, 1999). Para las cucurbitáceas es recomendado que se trasplante las plántulas con tres semanas de edad y dos hojas (Borne, 1999), ya que son plantas que poseen un desarrollo rápido. En pepino, Seabra Júnior et al. (2004) observaron reducción de la productividad con la utilización de plántulas viejas. Según Belfort y Gomes (2000), la edad de trasplante en que las plántulas son trasplantadas puede perjudicar su calidad. El volumen de la celda utilizado puede no ser compatible con el tiempo de permanencia de las plántulas en la bandeja y restringir el desarrollo de la raíz. Para los productores de plántulas, hay la tendencia de vender plántulas más nuevas, para reducir su tiempo en el vivero de producción. Los productores que van a cultivar estas plántulas prefieren los más desarrollados. Probablemente, esa preferencia está relacionada con la facilidad de trasplante, porque su sistema de radical compacto no se rompe en el momento de retirada de las bandejas (Seabra Júnior et al., 2004). Por lo antes expuesto, el objetivo de esta investigación fue estudiar el efecto del tipo de bandeja y edad de las plántulas en el rendimiento del cv. "Poinsett $76 "$.

\section{Materiales y métodos}

Las plántulas fueron producidas en ambiente protegido, en una estructura tipo capilla, con dimensiones de 4 x $10 \mathrm{~m} \mathrm{y}$ pie directo de $2,5 \mathrm{~m}$, cobertura de cristal y lateralmente sin tela antiáfidos. Para la siembra fueron utilizadas bandejas de poliestireno expandido con 72, 128, 162 y 200 celdas, correspondientes a un volumen de $52,25,18$ y $11 \mathrm{~cm}^{3}$, respectivamente. Estas fueron rellenadas con sustrato comercial Turba (Sogemix PG-M), utilizándose una semilla por celda de la variedad "Poinsett 76" de la empresa Semillas Magna, la cual es moderadamente vigorosa y adaptable a diversas condiciones climáticas, de frutos monoicos, 19 × $6 \mathrm{~cm}$, de forma cilíndrica y color verde oscuro, con ciclo de 65 días a cosecha aproximadamente, introducidas al país hace 60 años y de alta aceptación por el consumidor venezolano. El diseño experimental utilizado fue de bloques completos al azar, con tres repeticiones en arreglo factorial, cuyo primer factor fue cuatro tipos de bandejas (TB): 72, 128, 162 y 200 celdas y el segundo factor, la edad trasplante de plántulas (ET): 7 y 14 días después de la siembra (dds). Las siembras fueron realizadas con una diferencia cada siete días (29/03/2011 y 5/04/2011), respectivamente, para ser realizado el trasplante de las plántulas de todos los tratamientos en el mismo día (12/5/2011) en el aérea experimental (campo) en hileras de $10 \mathrm{~m}$ de longitud. El espaciamiento utilizado fue de $1,0 \times 0,33 \mathrm{~m}$. El sistema de riego utilizado fue goteo con fertirrigación.

El experimento se ubicó en el sector de San Vicente, municipio Maturín, estado Monagas, Venezuela, a 944’37’" de latitud Norte y los 6315'59" de longitud Oeste, y altitud de $51 \mathrm{msnm}$. Según Holdridge citado por Gil et al. (2000), el clima de la región es del tipo Bosque Seco Tropical, caracterizado por presentar una estación lluviosa de mayo a diciembre y una estación seca de enero a abril, con una precipitación media anual de $1219,6 \mathrm{~mm}$, una temperatura media anual de $25,9{ }^{\circ} \mathrm{C}$, con una evapotranspiración potencial de $1372 \mathrm{~mm}$ y una evaporación de $1573 \mathrm{~mm}$. Textura del suelo franco arenosa con $\mathrm{pH} 4,7 \mathrm{y}$ contenido de materia orgánica de $1,2 \%$.

La cosecha de los frutos comenzó el 20 de junio, cosechándose todos los frutos que presentaron características comerciales. Se realizaron evaluaciones de largo, ancho, peso del fruto, número de frutos por planta, rendimiento de frutos por hectárea. Se realizaron un total de seis cosechas agrupadas para su análisis de la manera siguiente: cosecha precoz: primera y segunda, cosecha intermedia: tercera y cuarta, cosecha tardía: quinta y sexta y cosecha total: la sumatoria de la primera, segunda, tercera, cuarta, quinta y sexta.

\section{Caracteres evaluados}

Los datos fueron recolectados del promedio de las plantas de las hileras centrales excluyendo los bordes de las hileras, y del promedio de 28 plantas seleccionadas por parcelas. El largo y ancho del fruto se tomó del promedio de diez frutos de cada cosecha tomados al azar y medidos con un vernier.

Para el peso del fruto se tomaron 10 frutos al azar en cada cosecha de cada tratamiento y se pesaron en una balanza de alta precisión. En la variable frutos por planta se contaron todos los frutos de 28 plantas de cada tratamiento y el total se dividió entre el número de plantas cosechadas y se tomó la media de los valores. Por último, el rendimiento 
$\left(\mathrm{t}^{*} \mathrm{ha}^{-1}\right)$ de frutos se calculó con el rendimiento obtenido de las veintiocho plantas de cada tratamiento en un área de 9,24 $\mathrm{m}^{2}$, después de eliminar los extremos o el efecto de bordura y mediante el cálculo de una regla de tres simple se estimó el rendimiento por hectárea.

\section{Análisis estadísticos}

Los datos se examinaron estadísticamente mediante un análisis de varianza y para la comparación de medias fue aplicada la prueba de rangos múltiple de Duncan. Todos los análisis estadísticos se realizaron con una probabilidad igual al $5 \%$ (Steel y Torrie, 1986). Para el análisis de los datos obtenidos en el experimento se usó el paquete estadístico SAS 9.0 (SAS Institute Inc., 2002).

\section{Resultados y discusión}

\section{Largo del fruto}

El largo y ancho del fruto es uno de los componentes de calidad que son atribuidos al tamaño y a la apariencia. El largo del fruto no fue afectado por la edad de las plántulas y tipo de bandeja variando de 17,11 a 17,25 y 16,96 a 17,47 $\mathrm{cm} ; 17,20$ a 17,33 y 17,02 a $17,63 \mathrm{~cm} ; 17,12$ a 17,26 y 16,94 a $17,47 \mathrm{~cm}$, en la cosecha precoz, intermedia, tardía, respectivamente (Tabla 1). Sin embargo, la interacción entre los factores edad de trasplante y tipo de bandeja, asimismo como los factores edad y bandeja, individualmente, fueron significativas en la cosecha total. Con la bandeja de 72 celdas $\left(52 \mathrm{~cm}^{3}\right)$ y $14 \mathrm{dds}$, se observaron los frutos con mayor longitud $(17,55 \mathrm{~cm})$, superior al obtenido en los demás tratamientos, pero diferencia estadística a los frutos producidos por las plántulas provenientes de bandeja de 52 $\mathrm{cm}^{3}$ y 7 dds (Tabla 2). Los frutos de menor longitud $(16,85$ $\mathrm{cm}$ ) a partir de las plántulas procedentes de bandeja de 200 celdas $\left(11 \mathrm{~cm}^{3}\right)$ y $14 \mathrm{dds}$.

Tabla 1. Promedios del largo del fruto de pepino (Cucumis sativus L.) cv. "Poinsett 76" en la cosecha precoz, intermedia, y tardía, en función del tipo de bandeja $(72,128,162$ y 200 celdas) y de la edad de trasplante (7 y 14 dds)

\begin{tabular}{|c|c|c|c|}
\hline \multirow{2}{*}{$\begin{array}{c}\text { TB } \\
\text { (celdas) }\end{array}$} & \multicolumn{3}{|c|}{ Largo $(\mathrm{cm})$ del fruto $\underline{1} /$} \\
\hline & $\begin{array}{c}\text { Cosecha } \\
\text { precoz }\end{array}$ & $\begin{array}{l}\text { Cosecha } \\
\text { intermedia }\end{array}$ & $\begin{array}{c}\text { Cosecha } \\
\text { tardía }\end{array}$ \\
\hline 72 & $17,47 \mathrm{a}$ & $17,63 \mathrm{a}$ & $17,47 \mathbf{a}$ \\
\hline 128 & $17,25 \mathrm{a}$ & $17,22 \mathrm{a}$ & $17,26 \mathbf{a b}$ \\
\hline 162 & $17,05 \mathrm{a}$ & $17,20 \mathrm{a}$ & $17,08 \mathbf{b}$ \\
\hline 200 & $16,96 \mathrm{a}$ & $17,02 \mathrm{a}$ & $16,94 \mathbf{b}$ \\
\hline C.V. $(\%)$ & 1,86 & 2,05 & 1,71 \\
\hline $\begin{array}{l}\text { ET } \\
\text { (dds) }\end{array}$ & \multicolumn{3}{|c|}{ Largo $(\mathrm{cm})$ del fruto $\underline{1} /$} \\
\hline 7 & $17,25 \mathrm{a}$ & $17,33 \mathrm{a}$ & $17,26 \mathrm{a}$ \\
\hline 14 & $17,11 \mathrm{a}$ & $17,20 \mathrm{a}$ & $17,12 \mathrm{a}$ \\
\hline C.V. $(\%)$ & 1,86 & 2,05 & 1,71 \\
\hline
\end{tabular}

1/Medias seguidas por la misma letra no difieren entre si estadísticamente por la prueba de Duncan a $5 \%$ de probabilidad.
Tabla 2. Efecto de la interacción en el largo promedio del fruto de pepino (Cucumis sativus L.) cv. "Poinsett 76 " en la cosecha total, en función de las diferentes edades de trasplante ( 7 y 14 dds) y tipo de bandeja (72, 128, 162 y 200 celdas)

\begin{tabular}{ccccc}
\hline \multirow{2}{*}{ ET } & \multicolumn{4}{c}{ Largo $(\mathrm{cm})$ del fruto $1 /$} \\
\\
\cline { 2 - 4 }$(\mathrm{dds})$ & 72 & 128 & 162 & \\
& $17,50 \mathrm{Aa}$ & $17,25 \mathrm{Ab}$ & $17,28 \mathbf{A b}$ & $17,11 \mathbf{A b}$ \\
7 & $17,55 \mathrm{Aa}$ & $17,24 \mathrm{Ab}$ & $16,95 \mathbf{B c}$ & $16,85 \mathbf{B c}$ \\
\hline
\end{tabular}

C.V. (\%): 0,72\%. 1/Medias seguidas de la misma letra mayúscula en la columna (celdas) y de la misma letra minúscula en las filas (edad de trasplante), no diferencian entre sí mismo al $5 \%$ de la probabilidad para la prueba de rango múltiples de Duncan.

\section{Ancho del fruto}

Para esta variable, la interacción entre los factores edad de trasplante y tipo de bandeja no fue significativa, asimismo como el factor edad, individual en las cosechas precóz, intermedia, tardía, y cosecha total, donde el ancho del fruto varió entre 5,44 a $5,49 \mathrm{~cm}$ en la precoz, $5,51 \mathrm{~cm}$ en la intermedia, 5,47 a $5,52 \mathrm{~cm}$ tardía, y 5,49 a $5,50 \mathrm{~cm}$ en la cosecha total, y promediaron 5,$47 ; 5,51 ; 5,50$ y 5,49 $\mathrm{cm}$, respectivamente (Tabla 3 ). No obstante, diferencias significativas $(\mathrm{p} \leq 0,05)$ fueron observadas en el ancho del fruto a causa del tipo de bandeja en todas las cosechas (precóz, intermedia, tardía, y cosecha total). La bandeja de 72 celdas promovió el mayor valor promedio, ya que fueron cosechados frutos de ancho 6,$01 ; 5,96 ; 6,00$ y 6,01 $\mathrm{cm}$, en las respectivas cosechas, en todas las cosechas. Los frutos de menor ancho se obtuvieron en las plantas provenientes de bandeja de 200 celdas (Tabla 3).

Tabla 3. Promedios del ancho del fruto de pepino (Cucumis sativus L.) cv. "Poinsett 76" en la cosecha precoz, intermedia, tardía y total, en función de la edad de trasplante ( 7 y 14 dds) y del tipo de bandeja $(72,128,162$ y 200 celdas $)$

\begin{tabular}{|c|c|c|c|c|}
\hline \multirow[b]{2}{*}{$\begin{array}{c}\mathrm{TB} \\
\text { (celdas) }\end{array}$} & \multicolumn{4}{|c|}{$\begin{array}{l}\text { Ancho }(\mathrm{cm}) \mathrm{del} \\
\text { fruto } 1 /\end{array}$} \\
\hline & $\begin{array}{l}\text { Cosecha } \\
\text { precoz }\end{array}$ & $\begin{array}{l}\text { Cosecha } \\
\text { intermedia }\end{array}$ & $\begin{array}{c}\text { Cosecha } \\
\text { tardía }\end{array}$ & $\begin{array}{c}\text { Cosecha } \\
\text { total }\end{array}$ \\
\hline 72 & $6,01 \mathrm{a}$ & $5,96 \mathbf{a}$ & $6,00 \mathbf{a}$ & $6,01 \mathbf{a}$ \\
\hline 128 & $5,48 \mathrm{~b}$ & $5,48 \mathbf{b}$ & $5,48 \mathbf{b}$ & $5,48 \mathbf{b}$ \\
\hline 162 & $5,26 \mathrm{c}$ & $5,36 \mathbf{b c}$ & $5,36 \mathbf{b}$ & $5,32 \mathrm{c}$ \\
\hline 200 & $5,11 \mathrm{c}$ & $5,24 \mathbf{c}$ & $5,16 \mathrm{c}$ & $5,17 \mathbf{d}$ \\
\hline C.V (\%): & 2,75 & 2,25 & 2,16 & 1,47 \\
\hline $\begin{array}{l}\text { ET } \\
\text { (dds) }\end{array}$ & \multicolumn{4}{|c|}{$\begin{array}{l}\text { Ancho }(\mathrm{cm}) \text { del fruto } \\
\underline{1 /}\end{array}$} \\
\hline 7 & $5,49 \mathrm{a}$ & $5,51 \mathrm{a}$ & $5,47 \mathrm{a}$ & $5,49 \mathrm{a}$ \\
\hline 14 & $5,44 \mathrm{a}$ & $5,51 \mathrm{a}$ & $5,52 \mathrm{a}$ & $5,50 \mathrm{a}$ \\
\hline Promedios & 5,47 & 5,51 & 5,50 & 5,49 \\
\hline C.V. $(\%)$ : & 2,75 & 2,25 & 2,16 & 1,47 \\
\hline
\end{tabular}

1/Medias seguidas por la misma letra no difieren entre si estadísticamente por la prueba de Duncan a $5 \%$ de probabilidad. 
López-Elías et al. (2011b) evaluaron la producción y calidad de pepino bajo condiciones de invernadero obtuvieron diámetro del fruto, dentro de los estándares establecidos para pepino tipo americano, con un diámetro de 5,0 cm. Estos resultados concuerdan al ancho del fruto obtenidos en el presente trabajo, cuyo promedio en la cosecha precoz fue de $5,47 \mathrm{~cm}$, en la cosecha intermedia; $5,52 \mathrm{~cm}$ y de $5,55 \mathrm{~cm}$ en la cosecha tardía, respectivamente. Chacón et al. (2017) estudiaron tres genotipos de pepino partenocárpico tipo mini cultivados bajo condiciones de invernadero. Los resultados obtenidos se ubicaron dentro del rango establecido por otros autores, el diámetro del fruto varió entre 3,80 y 4,4 cm (Shaw et al., 2000). Mientras que, en ensayos de pepino en invernadero, otros investigadores obtuvieron un diámetro del fruto que osciló entre 2,64 y 2,77 cm (Hochmuth et al., 2004). Asimismo, en otro estudio se encontró un rango entre 2,84 y 3,51 mm para esta variable (Soleimani et al., 2009), y en otra investigación se obtuvieron valores entre 3,50 y $3,70 \mathrm{~cm}$ para esta variable (Jasso- Chaverria et al., 2005). Por otra parte, otros autores encontraron que el diámetro del fruto osciló entre 4,60 y $5,80 \mathrm{~cm}$ (Gómez-López et al., 2006). Estos inferiores son similares a los obtenidos en la presente investigación, y en otro estudio se obtuvo un valor de 4,90 cm para el diámetro del fruto (Galindo et al., 2014). Santi et al. (2013) en un estudio sobre la producción de pepino japonés 'Tsuyataro' obtuvieron en la cosecha total un diámetro del fruto promedio que vario de 3,4 a $3,5 \mathrm{~cm}$. Estos resultados son inferiores a los obtenidos en la presente evaluación. La diferencia en los resultados, podría deberse probablemente, al cultivar, manejo cultural, tipo de riego aplicado, plan de fertilización, la densidad de siembra utilizada, localidad y tipo de suelo en donde se llevó a cabo el estudio, o etapa en la cual se realizó la recolección de los frutos para su consumo o comercialización. Westwood citado por Montaño \& Méndez (2009) señala que el ancho del fruto depende a su vez de otros parámetros: zona cortical, pulpa y cavidad central. Estos tres caracteres tienen también una clara influencia en el peso del fruto, pero su mayor interés radica en que determina un aspecto importante de la calidad del fruto como es la relación de la parte comestible dentro del mismo. Varios factores influyen sobre el tamaño del fruto: polinización, condiciones climáticas durante la etapa inicial del desarrollo del fruto, relación hoja-fruto y las prácticas culturales. El tamaño definitivo del fruto depende de: (1) número de células presente en el fruto cuajado, (2) número de divisiones celulares que ocurre posteriormente, y (3) la extensión que las células alcanzan. Las divisiones celulares durante el estado inicial del crecimiento del fruto tienen una mayor influencia en el tamaño definitivo del fruto.

\section{Peso del fruto}

Para la variable peso del fruto, la interacción entre los factores edad de trasplante y tipo de bandeja no fue significativa, asimismo como los factores edad y bandeja, individualmente en las cosecha precóz, intermedia, y cosecha total, ya que el peso del fruto varió entre 286,7 a 332,2 g en la cosecha precóz, 282,0 a 333, 8 g en la intermedia, 293,5 a 317,0 g en la cosecha total; el promedio general fue de 308,$6 ; 309,2$; y 306,2 g, respectivamente. En la cosecha tardía, los frutos de menor peso $(266,3 \mathrm{~g})$ se obtuvieron en las plántulas provenientes de bandeja de 72 celdas, pero el resto de los tratamientos no mostraron diferencias significativas entre sí (Tabla 4). No se observaron diferencias significativas $(p \leq 0,05)$ a causa de la edad de trasplante en todas las cosechas (precóz, intermedia, tardía, y cosecha total). El peso del fruto osciló de 304,9 a 312,3 g en la cosecha precóz, 304,5 a 313,9 g en la intermedia, 298,5 a 300,8 g en la tardía y 305,9 a 306,8 $\mathrm{g}$ en la total, y promedios de 308,6; 309,2; 299,6 y 306,2 g, respectivamente (Tabla 4).

Tabla 4. Promedios del peso del fruto de pepino (Cucumis sativus L.) cv. "Poinsett 76" en la cosecha precoz, intermedia, tardía y total, en función del tipo de bandeja (72, 128, 162 y 200 celdas) y de la edad de trasplante (7 y 14 dds)

\begin{tabular}{|c|c|c|c|c|}
\hline \multirow{2}{*}{$\begin{array}{c}\mathrm{TB} \\
\text { (Celdas) }\end{array}$} & \multicolumn{4}{|c|}{ Peso $(\mathrm{g})$ del fruto $\underline{1 /}$} \\
\hline & $\begin{array}{l}\text { Cosecha } \\
\text { precoz }\end{array}$ & $\begin{array}{l}\text { Cosecha } \\
\text { intermedia }\end{array}$ & $\begin{array}{c}\text { Cosecha } \\
\text { tardía }\end{array}$ & $\begin{array}{c}\text { Cosecha } \\
\text { total }\end{array}$ \\
\hline 72 & $332,2 \mathrm{a}$ & $282,0 \mathrm{a}$ & $266,3 \mathbf{b}$ & $293,5 \mathrm{a}$ \\
\hline 128 & $312,0 \mathrm{a}$ & $295,5 \mathrm{a}$ & $308,0 \mathbf{a}$ & $305,2 \mathrm{a}$ \\
\hline 162 & $303,7 \mathrm{a}$ & $333,8 \mathrm{a}$ & $309,0 \mathbf{a}$ & $317,0 \mathrm{a}$ \\
\hline 200 & $286,7 \mathrm{a}$ & $325,5 \mathrm{a}$ & $315,2 \mathbf{a}$ & $309,0 \mathrm{a}$ \\
\hline Promedios & 308,6 & 309,2 & 299,6 & 306,2 \\
\hline C.V(\%).: & 9,51 & 13,21 & 7,49 & 7,21 \\
\hline ET (dds) & \multicolumn{4}{|c|}{ Peso (g) del fruto $\underline{1} /$} \\
\hline 7 & $304,9 \mathrm{a}$ & $313,9 \mathrm{a}$ & $298,5 \mathrm{a}$ & $305,9 a$ \\
\hline 14 & $312,3 \mathrm{a}$ & $304,5 \mathrm{a}$ & $300,8 \mathrm{a}$ & $306,8 \mathrm{a}$ \\
\hline Promedios & 308,6 & 309,2 & 299,6 & 306,2 \\
\hline C.V. (\%): & 9,51 & 13,21 & 7,49 & 7,21 \\
\hline
\end{tabular}

1/Medias seguidas por la misma letra no difieren entre si estadísticamente por la prueba de Duncan a $5 \%$ de probabilidad.

López-Elías et al. (2011b) evaluaron la producción $\mathrm{y}$ calidad de pepino bajo condiciones de invernadero encontraron que el peso del fruto, estuvo dentro de los estándares establecidos para pepino tipo americano, con un peso promedio de $330 \mathrm{~g}$. Mientras que, Chacón et al. (2017) evaluaron tres genotipos de pepino partenocárpico tipo mini cultivados bajo condiciones de invernadero. Los datos obtenidos coincidieron con los encontrados por otros autores, quienes al cultivar pepino tipo mini en invernadero, encontraron un rango de peso del fruto entre 190 y $330 \mathrm{~g}$ (Gómez-López et al., 2006), y también con los resultados de otro trabajo donde se compararon genotipos de pepino tipo mini cultivados en invernadero, y se obtuvo para el peso del fruto una variación entre 156 y $247 \mathrm{~g}$ en otoño, y entre 122 y $256 \mathrm{~g}$ (Shaw et al., 2000). Por otra parte, en evaluaciones de pepino en invernadero, de siete genotipos en Brasil, y se encontró que el peso promedio del fruto osciló entre 279,2 y 300,8 g (Cardoso, 2002). Los valores del peso del fruto similares a los obtenidos en este trabajo, cuyos promedios en la cosecha precoz fue de $308 \mathrm{~g}$, en la 
cosecha intermedia de $309 \mathrm{~g}$, de 299 g en la cosecha tardía y en la cosecha 306,2 g Antunes et al. (2014) estudiaron la respuesta de pepino africano a la fertilización nitrogenada y obtuvieron un peso promedio de $328,98 \mathrm{~g}^{*}$ fruto $^{-1}$, superior a los pesos promedios obtenidos en este estudio. Suniaga et al. (2008) encontraron peso de frutos desde 271 a $422 \mathrm{~g}$ en pepino variedad "Poinsett 76", valores que están dentro del rango obtenido en este trabajo. Marcano et al. (2012), encontraron valores inferiores a este ensayo en plantas de pepino cultivadas en diferentes localidades, el mayor peso lo encontró en la localidad de las Canoítas y los menores valores en la localidad de Sabaneta y La Estancia. Con valores promedios comprendidos entre 157,10 a 201,05 g Santi et al. (2013) en un estudio sobre de la producción de pepino japonés 'Tsuyataro' obtuvieron en la cosecha total un peso del fruto que vario de 212,1 a $228,3 \mathrm{~g}$ *fruto-1

\section{Número frutos por planta}

Para la variable número de frutos* planta $^{-1}$, la interacción entre los factores edad de trasplante y tipo de bandeja no fue significativa, asimismo como los factores edad y bandeja, individualmente en la cosecha precoz y tardía, en el factor tipo de bandeja, el número frutos* planta $^{-1}$ varió entre 44,19 a 48,59 en la cosecha precoz y de 37,98 a 43,98 en la cosecha tardía y con promedios de 46,02 y 40,00 frutos* planta $^{-1}$. respectivamente (Tabla 5). Diferencias significativas $(\mathrm{p} \leq$ $0,05)$ fueron observadas en el frutos* planta $^{-1}$ debido al tipo de bandeja en la cosecha intermedia y total. El tipo de bandeja de 72 celdas promovió el mayor valor en promedio en ambas cosechas, obteniéndose de 59,15 y 148,48 frutos* $^{*}$ planta $^{-1}$, respectivamente, el resto de tratamientos no difirieron entre sí (Tabla 5). El número de frutos* planta $^{-1}$ varió de 44,32 a 47,72 en la cosecha precoz; de 47,38 a 47,42 en la intermedia; de 37,55 a 42,45 en la tardía y de 129,24 a 137,59 en la cosecha total y promediaron 46,02 ; 47,40; 40,00 y 133,42; respectivamente (Tabla 5).

Los resultados de este estudio concuerdan con los obtenidos por Rao et al. (2004) y Arunkuma et al. (2011) en India, así como Golabadi et al. (2013) en Irán, sus investigaciones independientes donde se realizó señalaron que el número de frutos de pepino por planta proyecta el mayor efecto positivo sobre la producción total del fruto de pepino, indicando de que ese carácter es uno de los componentes más confiables para la selección de genotipos de pepino de alto rendimiento de frutos. Pero superiores a los obtenidos por López-Eías et al. (2011b) quienes evaluaron la producción y calidad de pepino bajo condiciones de invernadero, y encontraron que el numero de frutos* planta $^{-1}$ en el hibrido "Esparón", fue mayor respecto de "Camán" y "Modan", con 17,7 frutos*planta-1. Chacón et al. (2017) encontraron diferencias significativas entre los genotipos para el número de frutos de primera calidad y el número total de frutos* planta $^{-1}$. El genotipo "Katrina" presentó el mayor número de frutos*planta ${ }^{-1}$ $(21,97)$. Asimismo, en otra investigación se compararon seis genotipos tipo mini y se encontró que el rendimiento comercial osciló entre 27,6 y 36,7 frutos* planta $^{-1}$, y entre
34,3 y 66,8 frutos* planta $^{-1}$ (Soleimani et al., 2009). Sin embargo, otros investigadores encontraron en pepino cultivado en invernadero, un rendimiento total que osciló entre 24 y 31 frutos* $^{*}$ planta $^{-1}$ (Rahil \& Qanadillo, 2015). Asimismo, en otro trabajo se cultivó un genotipo de pepino monoico tipo japonés en invernadero, y se obtuvo una producción total de 27,64 frutos* planta $^{-1} \mathrm{y}$ una producción comercial de 16,01 frutos* planta $^{-1}$ (Nomura \& Cardoso, 2000). Santi et al. (2013) evaluaron la producción de pepino japonés 'Tsuyataro' obtuvieron en la cosecha total un promedio de 19,4 frutos*planta ${ }^{-1}$. Todos estos datos son inferiores a los obtenidos en el presente ensayo. La diferencia en los resultados, podría deberse probablemente, al cultivar, manejo cultural, tipo de riego aplicado, plan de fertilización, la densidad de siembra utilizada, localidad y tipo de suelo en donde se llevó a cabo el estudio, o etapa en la cual se realizó la recolección de los frutos para su consumo o comercialización. El número de frutos* planta $^{-1}$ podría ser considerado como índice de selección para alta producción (Veena et al., 2013). Además, encontraron una relación lineal entre caracteres del fruto y rendimiento por planta, sugieren que el método de selección de mejora del cultivo podría ser enfocado principalmente sobre características del fruto. Resultados similares han sido reportados para el número de frutos*planta ${ }^{-1}$ y largo del fruto por Arunkuma et al. (2011). Mientras que, Oliveira et al. (2010), observaron que a mayor densidad de plantación en pepino se tiene una disminución en el número de frutos* planta $^{-1}$, lo cual es el resultado de un menor crecimiento de la planta. Investigaciones realizadas por (Ortíz et al., 2009) consideraron que la variable número de frutos y el área foliar por planta son los caracteres más relacionados con el rendimiento por planta.

Tabla 5. Promedios del número de frutos*planta-de pepino (Cucumis sativus L.) cv. "Poinsett 76" en la cosecha precoz, intermedia, tardía y total, en función del tipo de bandeja

$(72,128,162$ y 200 celdas $)$ y de la edad de trasplante (7 y 14 dds).

\begin{tabular}{|c|c|c|c|c|}
\hline \multirow{2}{*}{$\begin{array}{c}\text { TB } \\
\text { (Celdas) }\end{array}$} & \multicolumn{4}{|c|}{$\begin{array}{c}\text { Numero de } \\
\text { frutos*planta }{ }^{-1} \underline{1} /\end{array}$} \\
\hline & $\begin{array}{l}\text { Cosecha } \\
\text { precoz }\end{array}$ & $\begin{array}{c}\text { Cosecha } \\
\text { intermedia }\end{array}$ & $\begin{array}{c}\text { Cosecha } \\
\text { tardía }\end{array}$ & $\begin{array}{c}\text { Cosecha } \\
\text { total }\end{array}$ \\
\hline 72 & $45,35 \mathrm{a}$ & $59,15 \mathbf{a}$ & $43,98 \mathrm{a}$ & $148,48 \mathbf{a}$ \\
\hline 128 & $45,95 \mathrm{a}$ & $44,42 \mathbf{b}$ & $38,25 \mathrm{a}$ & $128,63 \mathbf{b}$ \\
\hline 162 & $48,59 \mathrm{a}$ & $45,16 \mathbf{b}$ & $39,79 a$ & $133,53 \mathbf{b}$ \\
\hline 200 & $44,19 a$ & $40,89 \mathbf{b}$ & $37,98 \mathrm{a}$ & $123,03 \mathbf{b}$ \\
\hline promedios & 46,02 & 47,40 & 40,00 & 133,42 \\
\hline C.V. (\%): & 14,14 & 17,98 & 10,37 & 8,79 \\
\hline $\begin{array}{c}\text { ET } \\
\text { (dds) }\end{array}$ & \multicolumn{4}{|c|}{$\begin{array}{l}\text { Numero de } \\
\text { frutos* } \text { planta }^{-1} \underline{1} /\end{array}$} \\
\hline 7 & $47,72 \mathrm{a}$ & $47,42 \mathrm{a}$ & $42,45 \mathbf{a}$ & $137,59 a$ \\
\hline 14 & $44,32 \mathrm{a}$ & $47,38 \mathrm{a}$ & $37,55 \mathbf{b}$ & $129,24 \mathrm{a}$ \\
\hline Promedios & 46,02 & 47,40 & 40,00 & 133,42 \\
\hline C.V. (\%): & 14,14 & 17,98 & 10,37 & 8,79 \\
\hline
\end{tabular}

1/Medias seguidas por la misma letra no difieren entre si estadísticamente por la prueba de Duncan a $5 \%$ de probabilidad. 


\section{$\underline{\text { Rendimiento por planta }}$}

Para el rendimiento por planta de pepino, la interacción entre los factores edad de trasplante y tipo de bandeja no fue significativa, asimismo como los factores edad de trasplante en la cosecha precoz e intermedia, y el tipo de bandeja, individualmente en la cosecha precoz y tardía. El rendimiento de frutos* planta $^{-1}$ varió de 0,907 a $1,102 \mathrm{~kg}$ en la cosecha precoz y de 0,989 a $1,039 \mathrm{~kg}$ en la intermedia, y promedió 0,993 y $1,014 \mathrm{~kg}$, respectivamente (Tabla 6). Sin embargo, se observaron diferencias significativas $(\mathrm{p} \leq 0,05)$ en el rendimiento de las plantas que crecieron en los diferentes tipos de bandejas, en la cosecha precoz y tardía. El tipo de bandeja de 72 celdas promovió mayor rendimiento de frutos* planta $^{-1}$ en la cosecha intermedia y total. En la cosecha intermedia el mayor rendimiento lo presentaron las plántulas que crecieron en la bandeja de 72 celdas $\left(1,180 \mathrm{~kg}^{*}\right.$ planta $\left.^{-1}\right)$ y el resto de los tratamientos no mostraron diferencias significativas entre sí. En la cosecha total, el mayor rendimiento de frutos $\left(3,045 \mathrm{~kg}^{*}\right.$ planta $\left.^{-1}\right)$ de frutos por lo produjo en las plántulas que crecieron en bandeja de 72 celdas, aunque sin diferencias significativas con el obtenido de las plántulas que crecieron en la bandeja de 162 celdas $\left(2,913 \mathrm{~kg}^{*}\right.$ planta $\left.{ }^{-1}\right)$. El menor rendimiento de frutos $\left(2,660 \mathrm{~kg}^{*}\right.$ planta $\left.{ }^{-1}\right)$ en la cosecha total, se observó en las plántulas producidas en bandeja 200 celdas (Tabla 6).

Tabla 6. Promedios del rendimiento de frutos por planta de pepino (Cucumis sativus L.) cv. "Poinsett 76" en la cosecha precoz, intermedia, tardía y total, en función del tipo de bandeja $(72,128,162$ y 200 celdas) y de la edad de trasplante $(7$ y $14 \mathrm{dds})$

\begin{tabular}{|c|c|c|c|c|}
\hline \multirow{2}{*}{$\begin{array}{c}\mathrm{TB} \\
\text { (Celdas) }\end{array}$} & \multicolumn{4}{|c|}{ Rendimiento $\left(\mathrm{kg}^{*}\right.$ planta $\left.^{-1}\right)$ de frutos $\underline{1} /$} \\
\hline & $\begin{array}{c}\text { Cosecha } \\
\text { precoz }\end{array}$ & $\begin{array}{c}\text { Cosecha } \\
\text { intermedia }\end{array}$ & $\begin{array}{c}\text { Cosecha } \\
\text { tardía }\end{array}$ & $\begin{array}{c}\text { Cosecha } \\
\text { total }\end{array}$ \\
\hline 72 & $1,048 \mathrm{a}$ & $1,180 \mathbf{a}$ & $0,818 \mathrm{a}$ & $3,045 \mathbf{a}$ \\
\hline 128 & $0,997 \mathrm{a}$ & $0,920 \mathbf{b}$ & $0,831 \mathrm{a}$ & $2,747 \mathbf{b c}$ \\
\hline 162 & $1,044 \mathrm{a}$ & $1,016 \mathbf{b}$ & $0,862 \mathrm{a}$ & $2,913 \mathbf{a b}$ \\
\hline 200 & $0,882 \mathrm{a}$ & $0,942 \mathbf{b}$ & $0,836 \mathrm{a}$ & $2,660 \mathbf{c}$ \\
\hline Promedios & 0,993 & 1,014 & 0,837 & 2,841 \\
\hline C.V (\%): & 11,09 & 9,80 & 8,78 & 5,73 \\
\hline ET (dds) & \multicolumn{4}{|c|}{$\begin{array}{c}\text { Rendimiento } \\
\left(\mathrm{kg}^{*} \text { planta }^{-1}\right) \text { de frutos } \underline{1} /\end{array}$} \\
\hline 7 & $1,012 \mathrm{a}$ & $1,039 \mathrm{a}$ & $0,885 \mathbf{a}$ & $2,932 \mathbf{a}$ \\
\hline 14 & $0,973 \mathrm{a}$ & $0,989 a$ & $0,788 \mathbf{b}$ & $2,750 \mathbf{b}$ \\
\hline Promedios & 0,993 & 1,014 & 0,837 & 2,841 \\
\hline C.V $(\%):$ & 11,09 & 9,80 & 8,78 & 5,73 \\
\hline
\end{tabular}

1/Medias seguidas por la misma letra no difieren entre si estadísticamente por la prueba de Duncan a 5\% de probabilidad.

Musa et al. (2015), señalan que el peso de frutos* planta $^{-1}$ difirió significativamente entre las diferentes edades de las plántulas. El máximo peso de frutos* planta $^{-1}(884,31$ g) lo registro las plántulas de 60 días de edad, la cual fue estadísticamente similar a las de 45 días $(859,17 \mathrm{~g})$, pero significativamente superior a los demás tratamientos. Velazco (2005), evaluó tres cultivares de pepino y obtuvo un rango de 2,370 a 2,450 de $\mathrm{kg}^{*}$ planta ${ }^{-1}$, resultados que coinciden con los obtenidos en este trabajo, en la cual se obtuvo rendimientos de 3,045 $\mathrm{kg}^{*}$ planta $^{-1}$, en la bandeja de 72 celdas, y de $2,913 \mathrm{~kg}^{*}$ planta $^{-1}$, en la bandeja de 162 celdas, sin diferencias estadísticas entre sí. Con respecto a la edad de trasplante el mayor rendimiento en la cosecha total lo obtuvo las plántulas con $7 \mathrm{dds}$, con $2,932 \mathrm{~kg}^{*}$ planta $^{-1}$. Chacón \& Monge (2017) encontraron diferencias entre genotipos para el rendimiento total por planta. El genotipo "Katrina" obtuvo el mayor rendimiento por planta $(6,057$ $\mathrm{kg}^{*}$ planta $\left.^{-1}\right)$, lo cual está relacionado con el hecho de que también fue el que produjo el mayor número de frutos por planta. En ensayos de pepino en invernadero, en México, a una densidad de 3,33 plantas* $\mathrm{m}^{-2}$, se encontró un rango de rendimiento total entre 6,380 y $8,200 \mathrm{~kg}^{*}$ planta- $^{-1}$ (Barraza, 2012). En Brasil, se evaluó siete genotipos tipo "caipira" a una densidad de 2,0 plantas* $\mathrm{m}^{-2}$, y se obtuvo una producción comercial que varió entre 5,600 y 11,560 kg*planta-1 (Cardoso, 2002). Santi et al. (2013) en la evaluación de la producción de pepino japonés "Tsuyataro" obtuvieron en la cosecha total un rendimiento de $4,236 \mathrm{~kg}$ de frutos*planta ${ }^{-1}$. Estos resultados son superiores a los obtenidos en la presente evaluación. La diferencia en los resultados, podría deberse probablemente, al cultivar, manejo cultural, tipo de riego aplicado, plan de fertilización, la densidad de siembra utilizada, localidad y tipo de suelo en donde se llevó a cabo el estudio, o etapa en la cual se realizó la recolección de los frutos para su consumo o comercialización.

\section{Rendimiento $\left(\mathrm{t}^{*} \mathrm{ha}^{-1}\right)$ de frutos}

Para el rendimiento por hectárea, la interacción entre los factores edad de trasplante y tipo de bandeja no fue significativa, asimismo como en los factores edad de trasplante, individualmente en la cosecha precoz e intermedia, y en el tipo de bandeja con respecto a la cosecha tardía. Sin embargo, diferencias significativas $(\mathrm{p} \leq 0,05)$ fueron observadas en el rendimiento de frutos a causa del tipo de bandeja en la cosecha precoz, intermedia y total. El tipo de bandeja de 72 celdas promovió el mayor rendimiento de frutos en las tres cosechas. En la cosecha precoz, el menor rendimiento se obtuvo con las plantas provenientes de las bandejas de 200 celdas $\left(24,700 \mathrm{t}^{*} \mathrm{ha}^{-1}\right)$, el resto de los tratamientos no mostraron diferencias significativas entre sí. En la cosecha intermedia, el mayor rendimiento $(35,034$ $\left.\mathrm{t}^{*} \mathrm{ha}^{-1}\right)$ lo produjeron las plantas procedentes de bandejas de 72 celdas, mientras que el menor rendimiento se observó en las plantas de las bandejas de 128 celdas $\left(27,570 \mathrm{t}^{*} \mathrm{ha}^{-}\right.$ $\left.{ }^{1}\right)$. El mayor volumen de cosecha total, se obtuvo con las plantas producidas en bandeja de 72 celdas $\left(91,283 \mathrm{t}^{*} \mathrm{ha}^{-}\right.$ ${ }^{1}$ ), aunque estadísticamente similar al obtenido con plantas producidas en bandeja de 162 celdas $\left(87,330 \mathrm{t}^{*} \mathrm{ha}^{-1}\right)$. Las plantas producidas en bandeja de 128 y 200 celdas $(82,361$ y $79,738 \mathrm{t}^{*} \mathrm{ha}^{-1}$, respectivamente), tampoco mostraron diferencias significativas entre sí (Tabla 7).

En estudios realizados por López-Elías et al. (2011a) en la productividad y calidad de dos cultivares de pepino en respuesta de densidad de plantación encontraron el mayor rendimiento con la densidad de 20,000 plantas ha $^{-1}$, con 65,1 
$\mathrm{t}^{*} \mathrm{ha}^{-1}$, sin diferencias entre cultivares. López-Elías et al. (2011b) indicaron que el híbrido "Esparón" aunque produjo el mayor número de frutos*planta-1, estadísticamente no obtuvo mayor rendimiento, lo cual se debió, principalmente a que los frutos fueron cosechados antes de alcanzar la madurez fisiológica y como su peso cambia rápidamente de un día a otro, el rendimiento depende de la fecha de corte (Staub et al., 2009). El cultivar de pepino "Aodai", cuando es tutorado, alcanza rendimientos de 70 a 100 $\mathrm{t}^{*} \mathrm{ha}^{-1}$, en cambio que las variedades rastreras producen alrededor de 12 a $25 \mathrm{t}^{*} \mathrm{ha}^{-1}$ (Filgueira, 2008). Musa et al. (2015), encontraron que el rendimiento de frutos varió significativamente entre los tratamientos, con tendencia similar al peso de frutos* planta $^{-1}$. El rendimiento de frutos obtenido en las plántulas de 60 días de edad fue el más alto $\left(17,69 \mathrm{t} \mathrm{ha}^{-1}\right)$, la cual estuvo a la par con las plántulas de 45 días $\left(77,19 \mathrm{t} \mathrm{ha}^{-1}\right)$, mientras que ambos tratamientos produjeron significativamente mayor rendimiento de frutos en comparación con las plántulas de 30 días y la siembra directa. Diferentes autores (Hasandokht \& Nosrati, 2010; Orzolek, 2004), también reportaron que las hortalizas cultivadas de las plántulas más viejas producen rendimientos más altos. Estos resultados están también en consonancia con los encontrados por Choudhari et al. (2002). Sin embargo, según Liptay (1988), los rendimientos de las plántulas de diferentes edades son similares, a pesar del menor rendimiento de las plántulas jóvenes de pepino. Los resultados obtenidos por los autores mencionados anteriormente, son inferiores a los obtenidos en este estudio en la cosecha total que fue de $91,283 \mathrm{t}^{*} \mathrm{ha}^{-1}$, con el uso de bandeja de 72 celdas independientes de la edad de trasplante y de $87,906 \mathrm{t}^{*} \mathrm{ha}^{-1}$, y en la edad de trasplante (7 dds) independiente de tipo de bandeja utilizada, observándose también que superaron a la producción por hectárea nacional en 5,59 veces, en comparación con el rendimiento nacional reportado por Fedeagro (2016) para este cultivo. Veena et al. (2013) encontraron una relación lineal entre caracteres del fruto y rendimiento por planta y sugieren que el método de selección de mejora del cultivo podría ser enfocado principalmente sobre características del fruto. Rao et al. (2004) y Arunkuma et al. (2011), así como Golabadi et al. (2013) indicaron que el número de frutos de pepino por planta proyecta el mayor efecto positivo sobre la producción total del fruto de pepino, indicación de que ese carácter es uno de los componentes más confiables para la selección de genotipos de pepino de alto rendimiento de frutos. Las variedades de los cultivos en diferentes épocas del año o ambientes reaccionan de manera diferente dentro un rango de condiciones climáticas, características del suelo y prácticas técnicas (Singh y Ram, 2012). En los trópicos húmedos, caracterizados por condiciones de clima bimodal, la producción de pepino está ganando mayor atención y su cultivo durante las estaciones del año. Sin embargo, detalles sobre las respuestas del cultivo a las diferentes estaciones varían, por lo tanto, exige atención para mejorar su productividad.
Tabla 7. Promedios del rendimiento de frutos de pepino (Cucumis sativus L.) cv. "Poinsett 76" en la cosecha precoz, intermedia, tardía y total, en función del tipo de bandeja $(72,128,162$ y 200 celdas) y de la edad de trasplante (7 y 14 dds).

\begin{tabular}{ccccc}
\hline \multirow{2}{*}{$\begin{array}{c}\text { TB } \\
\text { Celdas) }\end{array}$} & \multicolumn{4}{c}{$\begin{array}{c}\text { Rendimiento }\left(\mathrm{t}^{*} \mathrm{ha}^{-1}\right) \mathrm{de} \\
\text { frutos } \underline{\mathrm{I}} /\end{array}$} \\
\cline { 2 - 5 } & $\begin{array}{c}\text { Cosecha } \\
\text { precoz }\end{array}$ & $\begin{array}{c}\text { Cosecha } \\
\text { intermedia }\end{array}$ & $\begin{array}{c}\text { Cosecha } \\
\text { tardía }\end{array}$ & $\begin{array}{c}\text { Cosecha } \\
\text { total }\end{array}$ \\
\hline 72 & $29,167 \mathbf{a}$ & $35,034 \mathbf{a}$ & $24,509 \mathrm{a}$ & $91,283 \mathbf{a}$ \\
128 & $27,900 \mathbf{a}$ & $27,570 \mathbf{c}$ & $24,920 \mathrm{a}$ & $82,361 \mathbf{b c}$ \\
162 & $28,833 \mathbf{a}$ & $30,443 \mathbf{b}$ & $25,839 \mathrm{a}$ & $87,330 \mathbf{a b}$ \\
200 & $24,700 \mathbf{b}$ & $28,230 \mathbf{b c}$ & $25,063 \mathrm{a}$ & $79,738 \mathbf{c}$ \\
\hline Promedios & 27,650 & 30,319 & 25,083 & 85,178 \\
\hline C.V (\%): & 7,06 & 6,90 & 5,54 \\
ET (dds) & \multicolumn{5}{c}{5,73} \\
7 & $28,063 \mathrm{a}$ & $30,986 \mathrm{a}$ & $26,530 \mathbf{a}$ & $87,906 \mathbf{a}$ \\
14 & $27,238 \mathrm{a}$ & $29,653 \mathrm{a}$ & $23,635 \mathbf{b}$ & $82,450 \mathbf{b}$ \\
\hline Promedios & 27,650 & 30,319 & 25,083 & 85,178 \\
\hline C.V $(\%):$ & 7,06 & 6,90 & 5,54 & 5,73 \\
\hline
\end{tabular}

1/Medias seguidas por la misma letra no difieren entre si estadísticamente por la prueba de Duncan a $5 \%$ de probabilidad.

\section{Largo (cm) del fruto}

López-Elías et al. (2011a) en estudios realizados de la productividad y calidad de dos cultivares de pepino en respuesta a la densidad de siembra encontraron que no influyó en la calidad del fruto; sin embargo, el cultivar "Cortéz" presentó mayor longitud que "Criollo". En lo referente a la calidad del fruto, el cultivar "Cortéz" presentó una longitud del fruto, promedio de $21,0 \mathrm{~cm}$, seguido por el cultivar Criollo, con 20,4 cm. Marcano et al. (2012) estudiaron el crecimiento y desarrollo del cultivo, encontraron una longitud promedio de $21,31 \mathrm{~cm}$. LópezElías et al. (2011b) evaluaron la producción y calidad de pepino bajo condiciones de invernadero obtuvieron longitud del fruto, dentro de los estándares establecidos para pepino tipo americano, con una longitud de $23,2 \mathrm{~cm}$.

Santi et al. (2013) en un trabajo sobre la evaluación de la producción de pepino japonés 'Tsuyataro' obtuvieron en la cosecha total una longitud del fruto promedio que vario de 24,5 a $25,1 \mathrm{~cm}$. Valores son superiores al obtenido en este ensayo, donde se encontró un largo promedio de los frutos en la cosecha precoz de $17,18 \mathrm{~cm}$, en la cosecha intermedia; $17,27 \mathrm{~cm}$, y de $17,19 \mathrm{~cm}$, en la cosecha tardía. Pero se ubican dentro del rango encontrado en un ensayo con seis genotipos de pepino tipo "Beit Alfa"(mini), cultivados en invernadero, en el cual la longitud del fruto varió entre 14,5 y $21,9 \mathrm{~cm}$ (Shaw et al., 2000). Sin embargo, en un trabajo sobre producción de pepino tipo mini en invernadero en Irán, se evaluaron 15 genotipos y se encontró que la longitud del fruto osciló entre 12,43 y 16,27 cm (Soleimani et al., 2009). En un ensayo donde se cultivaron 12 genotipos, y obtuvieron un rango entre 13,6 y $17,8 \mathrm{~cm}$ para esta variable (Hochmuth et al., 2004). Además, en un estudio donde se evaluó un genotipo, y se encontró que la longitud del fruto 
varió entre 14,4 y 16,4 cm (Gómez-López et al., 2006). Asimismo, en otra investigación, se evaluó un genotipo y obtuvieron que esta variable osciló entre 14,9 y 15,0 $\mathrm{cm}$ (Jasso-Chaverria et al., 2005). Todos estos resultados son inferiores a los obtenidos en el presente trabajo, lo que demuestra la diversidad existente entre los frutos de pepinos. Chacón et al. (2017) evaluaron el rendimiento y calidad de tres genotipos de pepino partenocárpico tipo mini cultivados bajo condiciones de invernadero. Los datos demostraron una amplia variabilidad entre genotipos en cuanto a longitud del fruto vario entre 17,83 a 18,41 $\mathrm{cm}$. Por otra parte, otros investigadores cultivaron una variedad de pepino en invernadero, y encontraron una longitud del fruto de 19,3 cm (Galindo et al., 2014), el cual son resultados superiores a los obtenidos en el presente ensayo. La diferencia en los resultados, podría deberse probablemente, al cultivar, manejo cultural, tipo de riego aplicado, plan de fertilización, la densidad de siembra utilizada, localidad y tipo de suelo en donde se llevó a cabo el estudio, o etapa en la cual se realizó la recolección de los frutos para su consumo o comercialización. (Achal et al., 1986) señala que el largo del fruto es considerado como una de las características que más influye en el rendimiento.

\section{Conclusiones}

En los resultados obtenidos, para las condiciones experimentales, se concluye que la producción total fue superior en las plántulas producidas en bandejas con mayor volumen de sustrato (52 y $25 \mathrm{~cm}^{3}$, respectivamente). Sin embargo, las plántulas producidas en menor volumen de sustrato presentaron menor rendimiento cuando ellas fueron más viejas (14 días). La edad influyo en el rendimiento total de frutos cuando las plántulas fueron trasplantadas a los 7 días después de la siembra. La edad no influyo sobre la longitud, diámetro, peso del fruto, número de frutos por planta en las plántulas producidas en volumen de sustrato mayor. La producción de plántulas de pepino en bandejas resulto en alta productividad (con media de 91,3 $\mathrm{t}^{*} \mathrm{ha}^{-1}$ ).

\section{Literatura citada}

Achal, S.; Lal, S.D.; Pant, C.E. 1986. Variability studies in chilli. Progressive Horticulture, 18(3-4): 270-272.

Antunes, G.; Sato, F.A.P.; Puiatti, M.; Cecon, P.R.; Da Silva, G.C.C. 2014. Produtividade e qualidade de frutos de pepino africano em resposta à adubação nitrogenada. Rev. Ceres, Viçosa 61 (1): 141-146.

Arunkuma, K.H.; Ramanjinappa, V.; Hugar, A. 2011. Association of yield and yield components in F2 population of cucumber (Cucumis sativus L.). Plant Archives, 11(1): 457-459.

Barraza, F.V. 2012. Acumulación de materia seca del cultivo de pepino (Cucumis sativus L.) en invernadero. Temas Agrarios, 17(2): 18-29.

Belfort, C.C. \& Gomes, M.S.F. 2000. Avaliação da idade de transplantio para mudas de melancia. Horticultura Brasileira, 18: 468-469.
Borne, H.R. 1999. Produção de mudas de hortaliças. Guaíba: Agropecuária. 187 pp.

Cardoso, A.I.I. 2002. Avaliação de cultivares de pepino tipo 'caipira' sob ambiente protegido em duas épocas de semeadura. Bragantia (Campinas), 61(1): 43-48.

Chacón, P.K. \& Monge, P.J.E. 2017. Rendimiento y calidad de pepino (Cucumis sativus L.) cultivado bajo invernadero, 17(29) : 39-48.

Choudhari, S.M.; More, T.A. and Tazuke, A. 2002. Fertigation, fertilizer and spacing requirement of tropical gynoecious cucumber hybrids. Acta Horticulture, 588: 233-240.

Fedeagro. 2017. Estadísticas Agrícolas. Venezuela, VE. Disponible en: http://www.fedeagro.org/produccion/ default.asp. Filgueira, F. A. R. 2008. Novo manual de olericultura: Agrotecnologia moderna na produção e comercialização de hortaliças. Viçosa: UFV. 421p.

Gil, J.; Montaño, N.; Khan, L.; Gamboa, A. y Narváez, E. 2000. Efecto de diferentes estrategias de riego en el rendimiento y la calidad de dos cultivares de Melón (Cucumis melo L.) Bioagro, 12(01): 25-30.

Galindo, F. V.; Fortis, M.; Preciado, P. P.; Trejo, R.; Segura, M.A.; Orozco, J.A. 2014. Caracterización físicoquímica de sustratos orgánicos para producción de pepino (Cucumis sativus L.) bajo sistema protegido, Revista Mexicana de Ciencias Agrícolas, 5(7): 12191232.

Golabadi, M.; Eghtedary, A.R. and Golkar, P. 2013. Determining relationships between different horticultural traits in (Cucumis sativus L.) Genotypes with multivariate analysis. Sabrao Journal of Breeding and Genetics, (45)3: 447-457.

Gómez-López, M.D.; Fernández-Trujillo, J.P.; Baille, A. 2006. Cucumber fruit quality at harvest affected by soilless system, crop age and preharvest climatic conditions during two consecutive seasons, Scientia Horticulturae, 110: 68-78.

Hasandokht, M. R.; Nosrati, S. Z. 2010. Effect of transplant age and fruit pruning on earliness and total yield of greenhouse cucumber (Cucumis sativus L. cv. Sultan). Plant Ecophysiology, 2: 21-25.

Hochmuth, R.C.; Davis, L.L.L.; Laughlin, W.L.; Simonne, E.H.; Sargent, S. A.; and Berry, A. 2004. Evaluation of twelve greenhouse mini cucumber (Beit Alpha) cultivars and two growing systems during the 20022003 winter sea- son in Florida 2004. Disponible en: https://doi.org/10.17660/ActaHortic.2004.659.61

Jasso-Chaverria, C.; Hochmuth, G.J.; Hochmuth, R.C. and Sargent, S. A. 2005. Fruit yield, size, and color responses of two greenhouse cucumber types to nitrogen fertilization in perlite soilless culture, Hort Technology, 15(3): 565-571.

Liptay, A. 1988. Field survival and establishment of tomato transplants of various age and size. Acta Horticulture, 220: 203-210. 
López-Elías, J.; Huez, L.M.A.; Pacheco, A.F.; Jiménez, L.J. y Preciado, F.F.A. 2011a. Productividad y calidad de dos cultivares de pepino en respuesta a la densidad de plantación. Revista de Ciencias Biológicas y de la Salud. Biotecnia, XIII (1): 23-28.

López-Elías, J.; Rodríguez, J. C.; Huez, L. M. A.; Garza, O. S.; Jiménez, L. J. y Leyva, E. E.I. 2011b. Producción y calidad de pepino (Cucumis sativus L.) bajo condiciones de invernadero usando dos sistemas de poda. IDESIA (Chile), 29(2): 21-27.

Marcano, C.; Acevedo, I.; Contreras J.; Jiménez O.; Escalona, A. y Pérez, P. 2012. Crecimiento y desarrollo del cultivo pepino (Cucumis sativus L.) en la zona hortícola de Humocaro bajo, estado Lara, Venezuela. Revista Mexicana de Ciencias Agrícolas, 3 (8): 16291636.

Minami, K. 1995. Produção de mudas de alta qualidade em horticultura. São Paulo: T. A. Queiroz. 138 p.

Montaño, M.N.J. \& Méndez, N.J.R. 2009. Efecto del ácido indol-3-acético y el ácido naftalenacético sobre el largo y ancho del fruto de melón (Cucumis melo L.) cultivar “Edisto 47”. Revista UDO Agrícola, 9(3): 530-538.

Musa, M.; Jellani, G.; Atif, M.J.; Ullah, H. and Ali, M. 2015. Influence of seedling age on cucumber (Cucumis sativus L.) Production. SAARC J. Agri., 13(2): 214221.

Nesmith, D.S. and Duval, J.R. 1998. The effect of container size. HortTechnology, 8(4): 495-498.

Nomura, E.S. \& Cardoso, A.I.I. 2000. Redução da área foliar e o rendimento do pepino japonés. Scientia Agricola, 57(2): 257-261.

Oliveira, A.; Silva, J.; Oliveira, A.N.; Silva, D.; Santos, R.; Silva, N. 2010. Produção do maxixeiro em função de espaçamentos entre fileiras e entre plantas. Horticultura Brasileira, 8:344-347.

Ortiz, J.; Sánchez, F.; Mendoza, M.; Torres, A. 2009. Características deseables de plantas de pepino crecidas en invernadero e hidroponía en altas densidades de población. Rev. Fitotec. Mex. 32(4): 289-294.

Orzolek, M. 2004. Evaluating vegetable transplants. Vegetable, small fruit and specialty crops. Virginia Cooperative Extension, 3(3): 9.

Pereira, P.R.G. \& Martínez,H.E.P. 1999. Produção de mudas para o cultivo de hortaliças em solo e hidroponia. Informe Agropecuario, 20: 24-31.

Rahil, M.H.; Qanadillo, A. 2015. Effects of different irrigation regimes on yield and water use efficiency of cucumber crop. Agricultural Water Management, 148: $10-15$.

Rao, E.S.; Munshi, A.D.; Verma, V.K. 2004. Genetic association and interrelationship of yield and its components in cucumber (Cucumis sativus L.). Indian J. Hort. 61:315-318.

Santi, A.; Scaramuzza, W.L.M.P.; Soares, D.M.J.; Scaramuzza, J.F.; Dallacort, R.; Krause, W.; Tieppo,
R. C. 2013. Desempenho e orientação do crescimento do pepino japonês em ambiente protegido Horticultura Brasileira, 31: 649-653.

SAS [Statistical Analysis System]. 2002. The SAS System for Windows 9.0. User's Electronic Guide: Statiscs. SAS Institute, Inc. Cary, North Caroline. USA.

Seabra Júnior, S.; Gadum, J.; Cardoso, I. I. 2004. Produção de pepino em função da idade das mudas produzidas em recipientes com diferentes volumes de substrato. Horticultura Brasileira, 22: 610-613.

Shaw, N.L.; Cantliffe, D.J.; Rodríguez, J.C.; Taylor, S. and Spencer, D.M. 2000. Beit Alpha cucumber: an exciting new greenhouse crop. Proceedings of the Florida State Horticultural Society, 113: 247-253.

Singh, A. \& Ram, H.H. 2012. Estimates of stability parameters for yield and its components in cucumber (Cucumis sativus L.). Vegetable Sci. 39(1): 31-34.

Soleimani, A.; Ahmadikhah, A. and Soleimani, S. 2009. Performance of different greenhouse cucumber cultivars (Cucumis sativus L.) in southern Iran, African Journal of Biotechnology, 8(17): 4077-4083.

Staub, J. E.; Robbins, M.D. and Wehner, T.C. 2009. Cucumber. Cucurbit Breeding. Horticultural Science. North Carolina State University. 43p.

Steel, G.D.R. y Torrie, H. J. 1986. Bioestadística. Principios y Procedimientos. México, D.F.: McGraw-Hill.

Suniaga, J.; Rodríguez, A.; Rázuri, L.; Romero, E. y Montilla, E. 2008. Fertilización, mediante fertirriego, durante diferentes etapas del ciclo de cultivo del pepino (Cucumis sativus L.) en condiciones de bosque seco premontano. Agric. Andina. 15: 56-65.

Veena, R.; Singh, A.S.; Pitchaimuthu, M.; Souravi, K. 2013. Character association for fruit yield and yield traits in cucumber (Cucumis sativus L.). Electronic Journal of Plant Breeding, 4(1): 1108-1112.

Velazco, P. 2005. Estudio comparativo de tres densidades de siembra de un hibrido de pepino con dos clases de tutoreo. Tesis Ingeniero Agropecuario. Escuela Superior Politécnica Del Litoral. Ecuador. Disponible en: $\quad$ https://view.officeapps.live.com/op/view. aspx [consultado. 112p. 